\title{
Health professionals' opinions on supporting a cancer biobank: identification of barriers to combat biobanking pitfalls
}

\author{
Nicole J Caixeiro ${ }^{\star, 1,2,3}$, Hei Lan Byun ${ }^{4}$, Joseph Descallar ${ }^{2,5}$, Janelle V Levesque ${ }^{1,2,5,6}$, Paul de Souza ${ }^{1,2,3,4,5}$ \\ and Cheok Soon Lee $e^{1,2,3,4}$
}

\begin{abstract}
Although rarely acknowledged, a successful biobank is highly dependent on the support of the health professionals who assist the biobank in all aspects of its activities. In many cases, the lack of health professional support can be a limiting factor in the biobanking process of collecting and processing high-quality biospecimens. The aim of this study was to determine the attitudes of health professionals towards cancer biobanking. Using a 5-point Likert scale questionnaire, important aspects of biobanking, including accrual, quality, knowledge, responsiveness, impact, access, trust, governance and accreditation, were investigated. In total, 95 of 124 health and medical practitioners who were approached participated in this study (77\% response rate). Health professionals in general supported the aims of biobanking with $56 \%$ of participants showing willingness to create a biobank and recruit donors (accrual), $85 \%$ understanding the importance in the storage and distribution of biospecimens (quality), $88 \%$ having an appreciation for the role of a biobank in furthering cancer research (knowledge), $70 \%$ showing awareness of the use of biospecimens in future research initiatives (responsiveness) and 73\% demonstrating support for a biobank with proper control, authority and credibility measures in place (governance and accreditation). Overall, provided that proper information about the activities of the biobank and researcher access was transparent, health professionals were very willing to support cancer biobanking. These findings may assist in developing strategies for the establishment and maintenance of biobanks and aid the implementation of more effective policies and procedures to embed biobanking into routine hospital practices.
\end{abstract}

European Journal of Human Genetics (2016) 24, 626-632; doi:10.1038/ejhg.2015.191; published online 2 September 2015

\section{INTRODUCTION}

Biobanks are an integral part of improving the delivery of health care. ${ }^{1}$ These large collections of representative biological materials and associated health information ${ }^{2}$ allow partnerships to be established between multiple health disciplines and professions in alliance with patient communities to increase research capacity and its application to emerging health issues. Ultimately, biobanks aim to contribute to health care by building and enabling research in daily clinical practice, which in turn allows the more rapid translation of findings for the clinical benefit of patients. ${ }^{3}$

Successful biobanking requires the input of patients and clinicians as well as the support of health-care organisations to effectively incorporate the necessary procedures into routine workflows. ${ }^{4}$ Numerous empirical studies have demonstrated the importance of understanding the attitude of the general public towards biobanking, which has led to the implementation of stringent ethical, legal and social policies that enhance public support, participation and ultimately the success of a biobank. ${ }^{5-7}$ Furthermore, it is now well recognised that successful biobanking relies heavily on the collection and processing of high-quality biospecimens; so much so that, national and international forums have been established to aid standardisation of biobanks and their activities for quality assurance and sustainability. ${ }^{1,8,9}$
A successful biobank is inevitably, however, also highly dependent on the critical support of the health professionals. Clinicians with diverse disciplinary backgrounds including physicians, surgeons, pathologists, radiologists, nurses and other medical staff are central to the biobanking process, ${ }^{4}$ assisting in all aspects of biobanking from consent to biospecimen collection to data accrual. Lack of health professional support at any point during this process can be a limiting factor, creating a bottleneck in the biobank's ability to collect and process high-quality biospecimens. Currently, there is little in the literature describing the barriers that hinder health professional support to biobanking. However, findings into the opinions of clinicians towards supporting research in general have demonstrated a lack of awareness of research activities, time and financial constraints, perceived difficulty with informed consent, effects on doctorpatient relationships and a perception of poor benefits relative to 'costs' of research as potential barriers hindering research support. ${ }^{10-12}$ In particular, a recurring barrier for clinicians is that 'duty of care' considerations are often seen to be in conflict with their patient's participation in research studies. ${ }^{11}$ These considerations necessarily override research goals directed towards increasing knowledge or improving future therapy via research ${ }^{10-12}$ and this results in a reduction in patient participation in research studies. ${ }^{10,13,14}$ It is therefore likely that these similar perceptions may be hindering health

${ }^{1}$ Centre for Oncology Education and Research Translation (CONCERT), Liverpool, NSW, Australia; ${ }^{2}$ Ingham Institute for Applied Medical Research, Liverpool, NSW, Australia; ${ }^{3}$ Discipline of Pathology, School of Medicine, University of Western Sydney, Liverpool, NSW, Australia; ${ }^{4}$ Department of Oncology, Liverpool Hospital, Liverpool, NSW, Australia; ${ }^{5}$ School of Medicine, University of New South Wales, Sydney, NSW, Australia; ${ }^{6}$ Psycho-Oncology Research Group, University of New South Wales, Sydney, NSW, Australia ${ }^{*}$ Correspondence: Dr N Caixeiro, Centre for Oncology Education and Research Translation (CONCERT), Ingham Institute for Applied Medical Research, 1 Campbell Street, Liverpool, 2170 NSW, Australia. Tel: +61 28738 4727; Fax: +61 29602 9441; E-mail: nicole.caixeiro@sswahs.nsw.gov.au

Received 8 December 2014; revised 17 June 2015; accepted 28 July 2015; published online 2 September 2015 
professional support to biobanking and its lack of integration in hospital settings. Few studies have examined the opinions of health professionals on issues surrounding biobanks ${ }^{4,15}$ and these have not been focused on how health professionals from all specialities perceive the role of a biobank and how they may support or hinder the engagement in biobanking activities. Hence, a comprehensive study of the attitudes of health professionals directly or indirectly related to biobanking warrants investigation.

To determine the attitudes of health professionals towards cancer biobanking, we investigated the opinions of research-active and inactive health professionals towards several aspects of biobanking, including accrual, quality, knowledge, responsiveness, impact, access, trust, governance and accreditation.

\section{MATERIALS AND METHODS}

The Centre for Oncology Education and Research Translation (CONCERT) Biobank was established in November 2012 (Sydney, New South Wales (NSW), Australia) to collect, process, store, retrieve and disseminate biological samples and health information for research projects following informed consent from patients diagnosed with cancer. Informed consent is obtained before surgery by the nursing, medical or biobank staff. Tissues for biobanking are examined within the anatomical pathology department of the participating hospital by pathology staff and tissues deemed in excess of diagnostic requirements are stored securely and anonymously by the biobank for future use by researchers nationally.

Before the commencement of this study, ethics approval (LNR/13/LPOOL/ 422) was obtained from the South Western Sydney Local Health District Human Research Ethics Committee. Written informed consent was waived as the study was deemed to be in the low/negligible risk category, with return of the survey sufficient to indicate consent to participate.

\section{Participants and recruitment}

The study cohort consisted of 95 health professionals working at Liverpool Hospital (Sydney, NSW, Australia), a CONCERT Biobank collection site, who encountered cancer patients during their routine work activities regardless of whether they were (i) aware of the CONCERT Biobank or (ii) had heard of biobanking in their daily work routine. Participants were identified through internal hospital directories and recruited within their departments or at multidisciplinary team meetings. Participants were given the option to complete the survey either online using the University of New South Wales (Sydney, NSW, Australia) Key Survey $(n=4 ; 4 \%)$ or in a pen-and-paper copy format $(n=91 ; 96 \%)$. Response rates were calculated based on the number of online and pen-and-paper copy surveys completed compared with the number of surveys that had been distributed.

\section{Survey development}

The survey was developed by performing a review of the literature using the search terms: 'biobank' and 'attitudes' or 'opinions' or 'public' or 'health professionals'; references cited from the literature found from the above searches were also consulted. A total of 22 studies were reviewed, with common themes identified as issues affecting the success of a biobank considered categories for further evaluation ${ }^{3,7,15-17}$ and were used to create novel questions for this study. The eight response categories and their definitions are described in Tables $1 \mathrm{a}$ and $1 \mathrm{~b}$.

\section{Data collection}

Demographic data including age, gender, religious affiliation, education and profession from each participant were collected. Participants who indicated that they had previously heard of biobanking were asked an open-ended question of what they perceived the role of a biobank to be. Responses that met the minimum definition of a biobank, 'A repository of biological materials and health information for use in medical research', ${ }^{2}$ were further grouped into three common themes of a biobank's perceived role: (i) storage facility, (ii) research facility and/or (iii) improving knowledge and clinical outcomes. In some instances responses overlapped between themes and hence the total number of responses may be greater than the total $N$ recruited to the study (95).

Participants were asked to rate their agreement with the survey statements using a 5 -point Likert scale: $1=$ strongly agree, $2=$ agree, $3=$ unsure, $4=$ disagree and $5=$ strongly disagree. ${ }^{18}$ Trust questions, asking participants to rate their trust in various institutions and health-care systems also used a 5-point Likert scale ranging from strongly trust to strongly distrust. For each category, the Likert scale responses were summed and then scaled to a score out of 30 to determine their association with demographic characteristics. For negative question structures, responses were reversed scored before being scaled.

\section{Statistical analyses}

Two sample $t$-tests were used to compare the scaled response scores to demographic characteristics, with a $P$-value of $\leq 0.05$ considered to be significant. Pearson correlations were calculated to identify correlations between the response categories. Cronbach's alpha was used as a measure of the internal consistency and reliability of the survey items grouped within the response categories. A Cronbach's alpha value of $\geq 0.70$ indicated a strong degree of reliability. The data analyses for this study were generated using SAS Enterprise Guide software, Version 6.1 of the SAS System for Windows (SAS Institute Inc., Cary, NC, USA).

\section{RESULTS}

\section{Demographic characteristics and awareness of biobanks}

The demographic characteristics of the participants are presented in Supplementary Table S1. In total, 95 of 124 health and medical practitioners approached from Liverpool Hospital participated in this study (77\% response rate). The majority of the cohort were Englishspeaking (74\%) females (73\%), predominately nurses $(61 \%)$ and comparatively young, with $39 \%$ of professionals aged under 35 years (Supplementary Table S1). Of note, $51 \%$ of participants had a familial history of cancer and of those, 64\% had not heard of cancer biobanking. However, approximately half $(51 \%)$ of those who had not heard of biobanking were open to receiving more information about a cancer biobank and its activities. Of the 36\% that had heard of biobanking, $68 \%$ had heard about biobanking in the workplace, while other sources included media outlets (9\%), course work (9\%) and conferences $(6 \%)$. Furthermore, participants who had heard of biobanking were asked to provide comment on the role of a cancer biobank (Table 2), with most participants $(n=22,65 \%)$ perceiving the biobank as a storage facility for biospecimens (eg, Participant 54, Nurse: 'Bank that stores biological specimens'), 21 participants (62\%) identified it as a research facility (eg, Participant 86, Allied Health Professional: 'Tissue removal for research'), and 9 (26\%) recognised the potential for improved knowledge and clinical outcomes (eg, Participant 14, Nurse: 'Help examine the behaviour of the different cancers and possibly find a treatment and cure').

\section{Opinions on supporting a cancer biobank}

Initial analyses demonstrated health professional support for biobanking in terms of the response categories of accrual (strongly agree-agree $56 \%$ ), quality (strongly agree-agree $85 \%$ ), knowledge (strongly agreeagree $88 \%$ ), responsiveness (strongly agree-agree $70 \%$ ) and governance and accreditation (strongly disagree-disagree 73\%; Table 1a). Statements relating to public benefit and cancer research (eg, 'I would want the participant to know their results from research' and 'A cancer biobank would be beneficial in providing information needed in the work to cure cancer'; Table 1a questions 6 and 9, respectively) were regarded positively. Participants exhibited the highest level of uncertainty concerning the statements ' $I$ do not have time to be involved in a Cancer Biobank' (unsure 47\%) and 'I would help recruit participants for a Cancer Biobank if I could access the samples' (unsure 49\%; Table 1a 


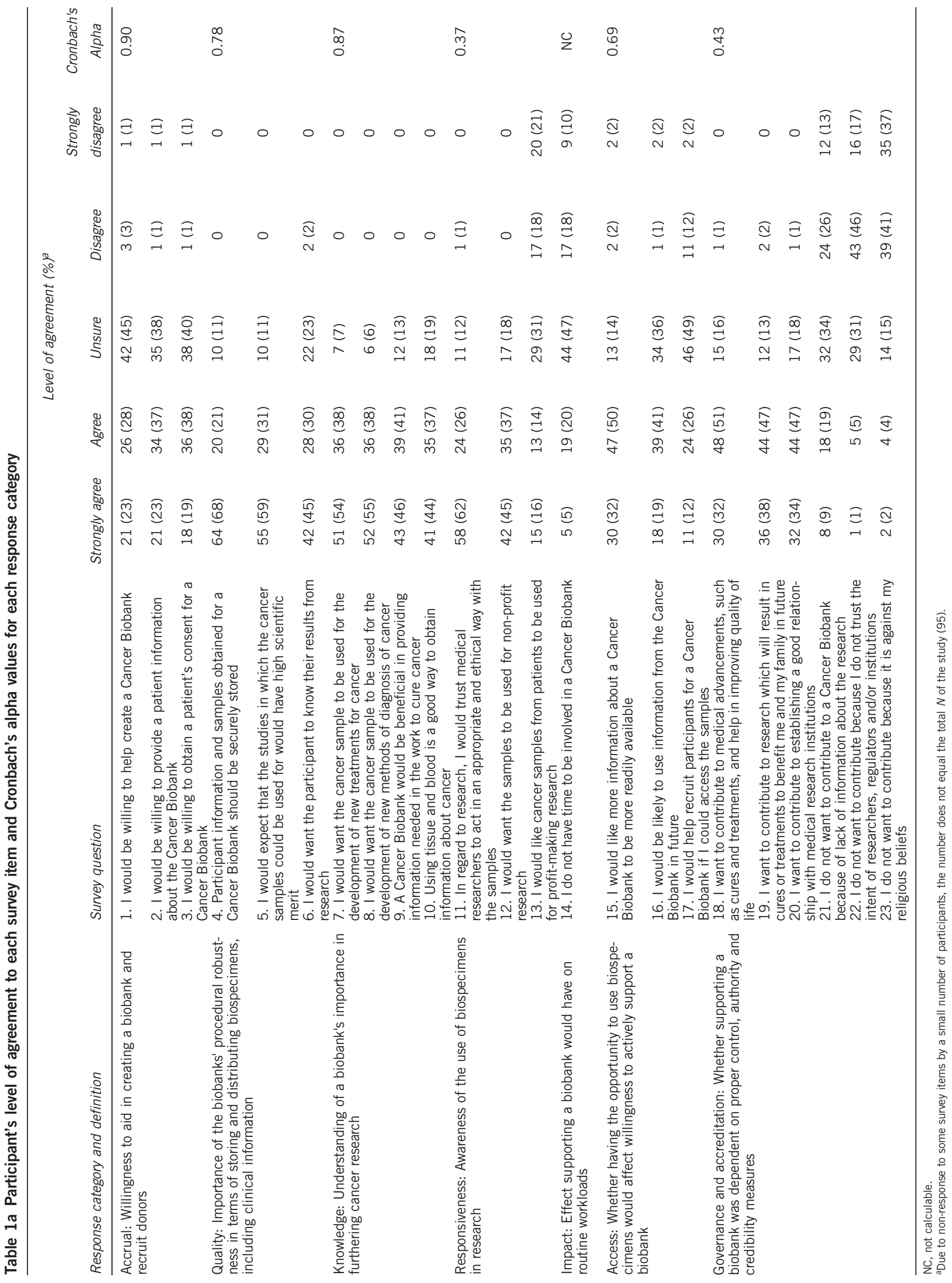


Table 1b Participant's level of trust to institutions and health-care systems

\begin{tabular}{llccccc}
\hline & & & \multicolumn{3}{c}{ Level of agreement (\%) } \\
Response category & Survey question & Strongly trust & Trust & Unsure & Distrust & Strongly distrust \\
\hline Trust & University research institutions & $18(19)$ & $53(56)$ & $20(21)$ & $3(3)$ & 0 \\
& Hospital research institutions & $23(24)$ & $55(59)$ & $16(17)$ & 0 & 0 \\
& For-profit company research institutions & $5(5)$ & $14(15)$ & $49(53)$ & $15(16)$ & $9(10)$ \\
& Government research institutions & $13(14)$ & $38(41)$ & $36(39)$ & $4(4)$ & $1(1)$ \\
\hline
\end{tabular}

aDue to non-response to some survey items by a small number of participants, the number does not equal the total $N$ of the study (95).

Table 2 Responses from participants who had previously heard of biobanking and their perceptions of the role of a cancer biobank

\begin{tabular}{|c|c|c|c|}
\hline $\begin{array}{l}\text { Participant number } \\
N=34 / 94^{a}\end{array}$ & Participant profession & Quotation & $\begin{array}{c}\text { Common biobanking } \\
\text { themes }\end{array}$ \\
\hline 1 & Administration Officer & Holding samples of tissue & i \\
\hline 2 & Technical Assistant/Officer & Collection of tissues and data for research purpose & ii \\
\hline 3 & Technical Assistant/Officer & Not sure, as only know a basic general idea of it & - \\
\hline 4 & Doctor & $\begin{array}{l}\text { Collecting clinical information and sections of tumours so that tissue samples can be } \\
\text { used for research to improve diagnosis and treatment of cancer }\end{array}$ & ii, iii \\
\hline 5 & Doctor & Build up a bank of tumour for future research & i, ii \\
\hline 6 & Doctor & $\begin{array}{l}\text { Preserving the tumour tissue for future research purposes and various treatment } \\
\text { modalities, testing for mutations, genetic analysis }\end{array}$ & i, ii, iii \\
\hline 7 & Doctor & Preserving tissue for current/future molecular/genetic research & i, ii \\
\hline 8 & Doctor & Increase knowledge base, especially genetics and molecular characteristics of tumours & iii \\
\hline 9 & Doctor & $\begin{array}{l}\text { Future retrieval of tissue and molecular cytogenetic studies if required and for research } \\
\text { purposes }\end{array}$ & i, ii \\
\hline 10 & Doctor & If would be helpful for future diagnosis and treatment of cancers & iii \\
\hline 11 & Administration Officer & Not sure, I do not know enough about it & - \\
\hline 12 & Administration Officer & Do not know enough about this & - \\
\hline 14 & Nurse & $\begin{array}{l}\text { Help examine the behaviour of the different cancers and possibly find a treatment and } \\
\text { cure }\end{array}$ & iii \\
\hline 16 & Doctor & Save pathological samples for studying & $\mathrm{i}, \mathrm{ii}$ \\
\hline 18 & Nurse & Collect samples for future study & $\mathrm{i}, \mathrm{ii}$ \\
\hline 24 & Nurse & Storing tissue & i \\
\hline 25 & Nurse & Genetic storage & $\mathrm{i}$ \\
\hline 27 & Doctor & Keep histopathology samples of tumour for study in future & $\mathrm{i}, \mathrm{ii}$ \\
\hline 30 & Nurse & Banking biological items such as bone marrow for when maybe needed later & $\mathrm{i}$ \\
\hline 38 & Nurse & Storage and management of samples for research & i, ii \\
\hline 47 & Doctor & To provide material for research and store for possible future use & i, ii \\
\hline 48 & Nurse & Tissue Bank for research purposes & ii \\
\hline 54 & Nurse & Bank that stores biological specimens & $\mathrm{i}$ \\
\hline 55 & Doctor & Collection of tissue samples for future research & i, ii \\
\hline 57 & Nurse & Keeping specimen for use transplant & - \\
\hline 59 & Nurse & $\begin{array}{l}\text { Storing of human biological samples for research and perhaps future use in scientific/ } \\
\text { medicine using data captured }\end{array}$ & i, ii, iii \\
\hline 68 & Allied Health Professional & Store biological tissue & $\mathrm{i}$ \\
\hline 70 & Nurse & $\begin{array}{l}\text { Biobank will store samples from cancer patients and use for research to help find a cure } \\
\text { for various types of cancer }\end{array}$ & i, ii, iii \\
\hline 71 & Nurse & Stores biological samples for use in research & i, ii \\
\hline 78 & Nurse & A place where tissue samples are stored and used for research & i, ii \\
\hline 82 & Allied Health Professional & $\begin{array}{l}\text { Collection of samples used for further research into cancer pathophysiology and } \\
\text { treatment }\end{array}$ & i, ii, iii \\
\hline 86 & Allied Health Professional & Tissue removal for research & ii \\
\hline 87 & Nurse & Bank a cancer specimen for future research & ii \\
\hline 89 & Nurse & $\begin{array}{l}\text { To collect blood and tissue samples from healthy individuals or patients diagnosed with } \\
\text { cancer or at risk of developing cancer including some information about health/treatment } \\
\text { of the donors. Samples/information available for research into causes/diagnosis/preven- } \\
\text { tion/treatments etc }\end{array}$ & i, ii, iii \\
\hline
\end{tabular}

i: storage facility; ii: research facility; iii: improving knowledge and clinical outcomes.

${ }^{a}$ Due to non-response to some survey items by a small number of participants, the number does not equal the total $N$ of the study (95). 
Table 3 Demographic variables impacting on health professionals' opinions

\begin{tabular}{|c|c|c|c|c|c|c|c|c|c|c|c|c|}
\hline \multicolumn{13}{|l|}{ Family history of cancer } \\
\hline Yes & 47 & 12.64 & 4.74 & 11.25 & 14.03 & $0.05^{b}$ & 48 & 6.53 & 2.33 & 5.86 & 7.21 & $0.04^{c}$ \\
\hline \multicolumn{13}{|l|}{ Heard of biobanking } \\
\hline
\end{tabular}

aDue to non-response to some survey items by a small number of participants, the number does not equal the total $N$ of the study (95). NB. The lower the mean value, the higher the agreement.

${ }^{b}$ Possible trend.

'Significant difference.

questions 14 and 17 , respectively), thereby suggesting the impact of biobanking on work-related activities and the opportunity to access samples could affect willingness to actively support a biobank. Collectively, these findings suggest that if greater knowledge about the use of samples and the information it provides to help cancer research were more readily available, that there could be more support to biobanking from health professionals. This coincides with the participants' strong agreement of understanding the biobank's important role in furthering cancer research (knowledge). In terms of the participants' perceived level of trust in various institutions and healthcare systems (Table 1b), hospital research institutions rated highest (strongly trust-trust $83 \%$ ) followed by university research institutions (strongly trust-trust 75\%) and government research institutions (strongly trust-trust 55\%). When asked to rate the level of trust of for-profit company research institutions, participants were unsure (53\%; Table 1b), thereby indicating health professionals are more likely to participate in biobanking if the biobank is affiliated with hospital, university and government research institutions and less likely with for-profit companies. However, participant's demonstrated that the level of uncertainty could also be interpreted as the participant's misunderstanding or lack of knowledge and experience pertaining to some survey items.

\section{Identification of issues to increase biobanking success}

To determine whether demographic variables impacted on health professionals' opinions on the various aspects of biobanking, survey questions were grouped within their categories and overall level of agreement for each response category calculated and compared with the demographic data. Negative question structures (governance and accreditation) were reverse scored when calculating the response category scores. The findings demonstrated no significant difference between the response categories of responsiveness, impact, access, trust as well as governance and accreditation to the demographic data, such as age, gender, religious affiliation, education and profession $(P>0.05)$. However, statistically significant differences were found between participants who had heard of biobanking than those who had not with the response category of accrual $(P=0.02)$ and a possible trend with knowledge $(P=0.06)$, that is, those who had heard of biobanking were in stronger agreement with aiding the biobank on a routine basis and recruiting donors (accrual) and understood the biobank's importance in furthering cancer research (knowledge; Table 3). A significant favourable difference was also observed between those participants with a familial history of cancer than those without with the response categories of knowledge $(P=0.04)$ and accrual $(P=0.05$; Table 3).
Comparison of whether there were any correlations between the level of agreement of one response category to another revealed the majority of categories moderately or weakly correlated with each other (Supplementary Table S2). Strong positive correlations were identified between the response categories of access and accrual $(r=0.64)$, and quality and knowledge $(r=0.69)$, thereby indicating those who strongly agreed with the importance of the biobank's procedural robustness of storing and distributing biospecimens (quality) also strongly agreed with its importance in furthering cancer research (knowledge). Interestingly, the response category of accrual had the highest number of strong to moderate associations, correlating with the response categories of quality, knowledge, impact, access and trust whereas impact inversely correlated with six of eight response categories: accrual, quality, knowledge, responsiveness, access and trust (Supplementary Table S2).

\section{Survey reliability}

Cronbach's alpha values were used to measure the reliability of the survey items (Table 1a). Overall, the survey showed good reliability with three of the six response categories (accrual, quality and knowledge) demonstrating Cronbach's alpha values $\geq 0.70$; and potentially a fourth response category, access, which had a Cronbach's alpha value of 0.69 (Table 1a). The response categories responsiveness and governance and accreditation reported low Cronbach's alpha values; 0.37 and 0.43 , respectively, which indicated a weak degree of intercorrelation between the single survey items and overall breath of the category. Optimising the number of items for these response categories may improve the reliability. The response category impact could not be assessed as there was only one survey item.

\section{DISCUSSION}

Although rarely acknowledged, a successful biobank is highly dependent on the support of the health professionals who inevitably assist the biobank in all aspects of its activities. In many cases, the lack of health professional support can be a limiting factor in the biobanking process and becomes a bottleneck in a biobank's ability to collect highquality biospecimens. In an effort to better understand the opinions of health professionals towards biobanking and identify barriers that may impede their involvement, this study developed a survey to provide empirical data to address issues of support from health professionals towards biobanking.

The study cohort surveyed was mostly female nurses with a modal age of under 35 years, which is reflective of the workforce at Liverpool Hospital. ${ }^{19}$ The few existing studies ${ }^{4,14-16}$ that previously examined medical practitioners' involvement in biobanking had primarily 
focused on clinician attitudes, whereas this study is the first to assess the responses of a broader cohort of health professionals. More importantly, this study examined the attitudes of nurses who, at present, have an integral part in liaising between hospital and biobank staff. Their opinions are, therefore, important when attempting to integrate biobanking into routine hospital practices, such as enrolling potential participants at pre-admissions clinic, discussing biobanking in perioperative communications, identifying participants at multidisciplinary team meetings and recording banked biospecimens in patient information and clinical notes.

This study revealed that despite approximately half of participants having a familial history of cancer, the large majority had not heard of biobanking. This surprising finding identified a clear need for more information about the biobank and its activities to be readily available within this health district. The finding was also directly supported by the identification of health professionals' strong desire for greater information about the biobank as well as the biobanks' procedural robustness in terms of storing and distributing biospecimens and information to be more available. Given the relative lack of awareness of the process of biobanking in this study cohort, it should be acknowledged that the health professionals' attitudes appear based on limited personal experience of biobanking and are thus hypothetical rather than factual. It will be of great interest to determine with further studies whether these views are altered once health professionals have more information about the process and are more actively involved in biobanking.

The data collected could not shed any light as to why the majority of health professionals had not heard of biobanking. Understanding this could assist in developing strategies to better disseminate relevant information to health professionals and subsequently to have biobanking implemented routinely into hospital workflows.

Overall, health professionals demonstrated a favourable response to biobanking as long as the activities of the biobank and those of the researchers accessing material were well documented and the information readily available to all participants. These findings are similar to those of Jenkins et a ${ }^{20}$ who also reported that up to $83 \%$ of patients would be willing to participate in cancer-related research if they were provided with complete information about the study requirements. ${ }^{20}$ Our findings demonstrated that medical professionals expressed strong opinions on the quality of the research and the purpose of the donated samples, which coincide with the findings of Luque et $a l^{21}$ whom, after surveying public attitudes to biobanking, found that the benefit of contributing to new cancer treatments through intrinsic medical research outweighed negative issues such as mistrust of research and privacy. There was also a strong desire to have cancer biobank participants informed of the result of any future research. These findings concur with several studies demonstrating the convergence of both public ${ }^{22}$ and researcher ${ }^{16}$ opinions that individual results should be disclosed to biobank participants. It has now been demonstrated by these findings that medical and health professionals also agree it is important for biobanks to disclose participant results. Currently, this is not common practice among biobanks as there is a controversial debate as to whether there is an ethical obligation for disclosure. This stems from multiple factors including public concern over privacy issues, compensation for the donated samples, and the potential for genetic discrimination. Moreover, the estimated number of disease-associated variants needed to be made available to meet the criteria of disclosure is between 11000 and 15000, which raises significant practical issues in providing reliable information in a format that is meaningful to all participants. There is also a particular concern with results that are experimental and for which the clinical value of knowing the information is uncertain. ${ }^{23,24}$ Ultimately, given the large and increasing amount of data generated from the application of new experimental technologies to biobanked samples, the preference for full disclosure of research results identified here suggests that the development of policies and mechanisms addressing the disclosure of information are important considerations if biobanking is to be embedded into routine clinical practice.

The findings of this study further demonstrated that health professionals familiar with what is involved in biobanking were willing to participate in its daily activities from consent to biospecimen collection. Moreover, despite some participants' uncertainty on the impact biobanking could have on routine workloads, there remained a strong favourable response to the other aspects of biobanking. These results refute previous suggestions that the lack of health professional support may have been due to a lack of dedicated time attributable to workload pressures and culture (practice-based attitudes) or due to clinicians concerns of how it would impact patients (patient-based attitudes). ${ }^{13,14,25}$ For instance, previous studies have identified practice-based attitudes in which clinicians expressed concerns about the lack of staff training and their previous experience in research, ${ }^{11,25}$ increased paperwork and administration ${ }^{11}$ and a perception that research involvement impedes clinical autonomy and may impact on the doctor-patient relationship. ${ }^{25}$ Similar to the findings of Ulrich et al, ${ }^{14}$ our results demonstrate that no such barriers impede health professionals support of biobanking activities. However, our findings are in contrast to research into the attitudes of clinicians towards clinical trials which demonstrated that health professionals act as bottlenecks in the accrual of participants to these research studies. ${ }^{10,13,14}$ In terms of patient-based attitudes, clinicians do not inform potential participants about research studies due to their own opinions and judgements of the patient's willingness to take part, ability to understand the research process or likelihood to adhere to the research protocol. ${ }^{10,13,14}$ Such attitudes, as demonstrated by Fallowfield et al, ${ }^{12}$ drastically reduce the number of participants approached for research by clinicians. Although, the findings of this study appear to be in contrast to these results, more direct evidence is needed. Further studies would include utilising the theory of planned behaviour to identify whether the attitudes of health professionals towards biobanking would predict their actual involvement. ${ }^{26}$

Despite the important findings of this study, there were several limitations. First, although there was an overrepresentation of nurses among the health professionals, which could overshadow the attitudes of other medical practitioners, this did not negate the fact that nurses are primary ports of call for patients as well as liaisons between hospital and biobank staff and are likely a core group to target biobanking interventions. However, further study would benefit from a larger sample size capturing a greater representative cohort of all the health professionals. Second, the ability to translate several key biobanking issues into survey questions is a limiting factor and survey validation would strengthen the findings of this study. While factor analysis was considered, the sample size in this study fell below the general sample size required for the number of survey items (minimum sample of $n=115$ ) to ensure adequate power in analysis. ${ }^{27}$ Instead, Cronbach's alpha was used as a measure of reliability and demonstrated three of the six categories, with a possible fourth category, were reliable. Despite this, optimising the number of items in each response category could more effectively capture the attitudes of practitioners towards biobanking and the reliability of the measures. For example, the 'impact' response category only contained one survey item and would benefit from the inclusion of additional assessment criteria such as the impact in terms of routine workflow, 
quality of research, and doctor-patient relationships. Further refinement of the scale may also see some survey items removed or placed under different response categories to improve both Cronbach's alpha values and the degree of inter-correlation between single survey items and overall breadth of the response categories. Furthermore, participants' understanding of survey items, where questions were marked as unsure, could signify misinterpretation rather than preference. Uncertainty in responses may also reflect uncertainty pertaining to the practical involvement in biobanking and how this would affect health professionals' workflows, as this study demonstrated a large majority had not heard of biobanking. These issues could be addressed in future studies by examining preferences to actual behaviour or performing semi-structured interviews.

\section{CONCLUSION}

A better understanding of the attitudes of health and medical professionals towards cancer biobanking has helped identify issues, which impede the successful integration of a biobank into routine hospital practices. The findings of this study demonstrate a variable understanding and knowledge about biobanking and its practices among medical and health professionals. Despite several limiting aspects of the study design, it is evident that a greater comprehension of biobanking and its activities could potentially lead to increased support. Furthermore, the identification of these issues and the subsequent development of strategies to address them are likely to be essential if translation of biobanking into routine clinical practice is to be achieved. The implementation of policies and procedures that aid health professionals' contribution to the biobank is an important first step in this process to facilitate the effective translation of this vital infrastructure tool for cancer research into clinical practice.

\section{CONFLICT OF INTEREST}

The authors declare no conflict of interest.

\section{ACKNOWLEDGEMENTS}

This work was supported by the University of Western Sydney and the University of New South Wales. Funding sources include the Cancer Institute New South Wales for CONCERT. We thank Norbert Kienzle and Jennifer O'Shea for their thoughtful contributions to the study and Björn Espedido and Kieran Scott for critical review of the manuscript.

1 Barnes RO, Parisien M, Murphy LC, Watson PH: Influence of evolution in tumor biobanking on the interpretation of translational research. Cancer Epidemiol Biomarkers Prev 2008; 17: 3344-3350.

2 Otlowski MFA, Nicol D, Stranger MJA: Biobanks information paper 2010. J Law Info Sci 2010; 20: 97-227.
3 Riegman PH, Morente MM, Betsou F, de Blasio P, Geary PMarble Arch International Working Group on Biobanking for Biomedical Research: Biobanking for better healthcare. Mol Oncol 2008; 2: 213-222.

4 Wyld L, Smith S, Hawkins NJ, Long J, Ward RL: Introducing research initiatives into healthcare: what do doctors think? Biopreserv Biobank 2014; 12: 91-98.

5 Nicol D, Critchley C: Benefit sharing and biobanking in Australia. Public Underst Sci 2012; 21: 534-555.

6 O'Doherty KC, Hawkins A: Structuring public engagement for effective input in policy development on human tissue biobanking. Public Health Genomics 2010; 13: 197-206.

7 Whitley EA, Kanellopoulou N, Kaye J: Consent and research governance in biobanks: evidence from focus groups with medical researchers. Public Health Genomics 2012; 15: 232-242.

8 Garcia DL, Bracci PM, Guevarra DM, Sieffert N: International Society for Biological and Environmental Repositories (ISBER) tools for the biobanking community. Biopreserv Biobank 2014; 12: 435-436.

9 Vaught J, Campbell LD, Betsou F et al: The ISBER best practices: insight from the editors of the third edition. Biopreserv Biobank 2012; 10: 76-78.

10 Ford E, Jenkins V, Fallowfield L, Stuart N, Farewell D, Farewell V: Clinicians' attitudes towards clinical trials of cancer therapy. Br J Cancer 2011; 104: 1535-1543.

11 Caldwell PH, Craig JC, Butow PN: Barriers to Australian physicians' and paediatricians' involvement in randomised controlled trials. Med J Australia 2005; 182: 59-65.

12 Fallowfield L, Ratcliffe D, Souhami R: Clinicians' attitudes to clinical trials of cancer therapy. Eur J Cancer 1997; 33: 2221-2229.

13 Grunfeld E, Zitzelsberger L, Coristine M, Aspelund F: Barriers and facilitators to enrollment in cancer clinical trials: qualitative study of the perspectives of clinical research associates. Cancer 2002; 95: 1577-1583.

14 Ulrich CM, James JL, Walker EM et al: RTOG physician and research associate attitudes, beliefs and practices regarding clinical trials: implications for improving patient recruitment. Contemp Clin Trials 2010; 31: 221-228.

15 Leiman DA, Lorenzi NM, Wyatt JC, Doney AS, Rosenbloom ST: US and Scottish health professionals' attitudes toward DNA biobanking. J Am Med Inform Assoc 2008; 15: 357-362.

16 Meulenkamp TM, Gevers SJ, Bovenberg JA, Smets EM: Researchers' opinions towards the communication of results of biobank research: a survey study. Eur J Hum Genet 2012; 20: 258-262.

17 Johnsson L, Helgesson G, Rafnar $T$ et al: Hypothetical and factual willingness to participate in biobank research. Eur J Hum Genet 2010; 18: 1261-1264.

18 Likert R: A technique for the measurement of attitudes. Arch Psychol 1932; 22: 55.

19 Harris P, Barton M: Research Strategy for South Western Sydney Local Health District 2012-2021. South Western Sydney Local Health District 2012. Available from http:// www.swslhd.nsw.gov.au/pdfs/SWSLHD_Research_Strategy_2012.pdf.

20 Jenkins V, Farewell D, Batt $L$ et al: The attitudes of 1066 patients with cancer towards participation in randomised clinical trials. $\mathrm{Br} J$ Cancer 2010; 103 : 1801-1807.

21 Luque JS, Quinn GP, Montel-Ishino FA et al: Formative research on perceptions of biobanking: what community members think. J Cancer Educ 2012; 27: 91-99.

22 Beskow LM, Smolek SJ: Prospective biorepository participants' perspectives on access to research results. J Empir Res Hum Res Ethics 2009; 4: 99-111.

23 Allen NL, Karlson EW, Malspeis S, Lu B, Seidman CE, Lehmann LS: Biobank participants' preferences for disclosure of genetic research results: perspectives from the OurGenes, OurHealth, OurCommunity project. Mayo Clin Proc 2014; 89: 738-746.

24 Cassa CA, Savage SK, Taylor PL, Green RC, McGuire AL, Mandl KD: Disclosing pathogenic genetic variants to research participants: quantifying an emerging ethical responsibility. Genome Res 2012; 22: 421-428.

25 Ross S, Grant A, Counsell C, Gillespie W, Russell I, Prescott R: Barriers to participation in randomised controlled trials: a systematic review. J Clin Epidemiol 1999; 52: 1143-1156.

26 Ajzen I: The theory of planned behaviour: reactions and reflections. Psychol Health 2011; 26: 1113-1127.

27 Allen P, Bennett K: PASW Statistics by SPSS: A Practical Guide Vol. 18South Melbourne: Cengage Learning, 2010.

Supplementary Information accompanies this paper on European Journal of Human Genetics website (http://www.nature.com/ejhg) 